\title{
Pupil Diameter as a Measure of Emotion and Sickness in VR
}

\author{
Brendan John \\ Department of Computer and Information Science and Engineering \\ University of Florida \\ brendanjohn@ufl.edu
}

\begin{abstract}
Eye tracking is rapidly becoming popular in consumer technology, including virtual and augmented reality. Eye trackers commonly provide an estimate of gaze location, and pupil diameter. Pupil diameter is useful for interactive systems, as it provides means to estimate cognitive load, stress, and emotional state. However, there are several roadblocks that limit the use of pupil diameter. In VR HMDs there are a lack of models that account for stereoscopic viewing and the increased brightness of near eye displays. Existing work has shown correlations between pupil diameter and emotion, but have not been extended to VR environments. The scope of this work is to bridge the gap between existing research on emotion and pupil diameter to VR, while also attempting to use pupillary data to tackle the problem of simulator sickness in VR.
\end{abstract}

\section{CCS CONCEPTS}

- Human-centered computing $\rightarrow$ Virtual reality; User studies; Interactive systems and tools; • Applied computing $\rightarrow$ Psychology;

\section{KEYWORDS}

Pupil Diameter, Virtual Reality, Pupillary Light Reflex, Arousal Classification, Simulator Sickness

ACM Reference Format:

Brendan John. 2019. Pupil Diameter as a Measure of Emotion and Sickness in VR. In 2019 Symposium on Eye Tracking Research and Applications (ETRA '19), June 25-28, 2019, Denver, CO, USA. ACM, New York, NY, USA, 3 pages. https://doi.org/10.1145/3314111.3322868

\section{INTRODUCTION}

The human eye provides a wealth of information regarding the individual, and many say it serves as a window into the soul. It is well documented that pupil diameter can be used to reveal information regarding cognitive load [Beatty 1982; Krejtz et al. 2018; Marshall 2002], task performance [White and French 2017], emotional arousal [Bradley et al. 2008], and stress [Pedrotti et al. 2014]. Despite a large body of work establishing pupil diameter as an index of cognitive load and emotional state, this modality is not commonly integrated into sensor suites. The scope of this work is to establish pupil diameter as a method of measuring emotional arousal and simulator sickness induced by VR displays.

Permission to make digital or hard copies of part or all of this work for personal or classroom use is granted without fee provided that copies are not made or distributed for profit or commercial advantage and that copies bear this notice and the full citation on the first page. Copyrights for third-party components of this work must be honored.

For all other uses, contact the owner/author(s).

ETRA '19, June 25-28, 2019, Denver, CO, USA

(C) 2019 Copyright held by the owner/author(s)

ACM ISBN 978-1-4503-6709-7/19/06.

https://doi.org/10.1145/3314111.3322868

\section{RESEARCH OBJECTIVES}

To generate research objectives I will first identify roadblocks that confound the use of pupil diameter in current VR displays. First, note that the vast majority of prior research in pupil diameter applications have been performed on traditional 2D displays. These displays present content at a single depth. VR HMDs display immersive $3 \mathrm{D}$ content to the user with binocular viewing. As a result these displays create a vergence/accommodation conflict, where the eyes converge to a depth other than that of the display.

Second, it is well established that the brightness of light entering the eye triggers a response in pupil diameter with a short latency [De Groot and Gebhard 1952; Reeves 1918; Young and Biersdorf 1954]. Pupils constrict to a smaller size with bright light, and dilate to a larger size in dark environments. This response should be removed from the signal for accurate classification.

Third, there is a need for a simple model that would allow for pupil diameter to replace established measures. Ground truth emotional response and simulator sickness is most commonly found through self reports [Bradley and Lang 1994; Kennedy et al. 1993] or real time annotation [Cowie et al. 2000; Mavridou et al. 2018]. Currently the types of sensors most strongly correlated with ground truth include EEG, HRV, and GSR. Pupil diameter is difficult to correlate with these established sensors, in part because of the one dimensional of data being produced. Based on these open issues within the field, my research objectives are as follows:

- $R_{1}$ : Validate the use of existing pupillary light response models within a stereoscopic VR display. This is necessary to apply pupil diameter as a measure in VR environments.

- $R_{2}$ : Establish a correlation between pupil diameter and existing measures as an index of emotion and simulator sickness in VR environments. This is necessary to show pupil diameter is an alternative to more complex and invasive sensors.

\section{HYPOTHESIS AND PROBLEM STATEMENT}

My research goals can be split into two components. The first component would require successfully modeling pupillary light reflex (PLR) in VR. Second, there is a need to evaluate models that classify emotion or simulator sickness based on changes in pupil diameter of the user. Formally my hypotheses are as follows:

- $H_{1}$ : The calibration procedure and application of a PLR model at a fixed depth in VR produces errors at the same scale as traditional 2D displays.

- $H_{2}$ : The calibration procedure and application of a PLR model at multiple depths in VR produces errors at the same scale as traditional 2D displays.

- $H_{3}$ : A light compensated PLR model shows significant correlation with emotional arousal and valence in VR.

- $H_{4}$ : A light compensated PLR model shows significant correlation with simulator sickness in VR. 

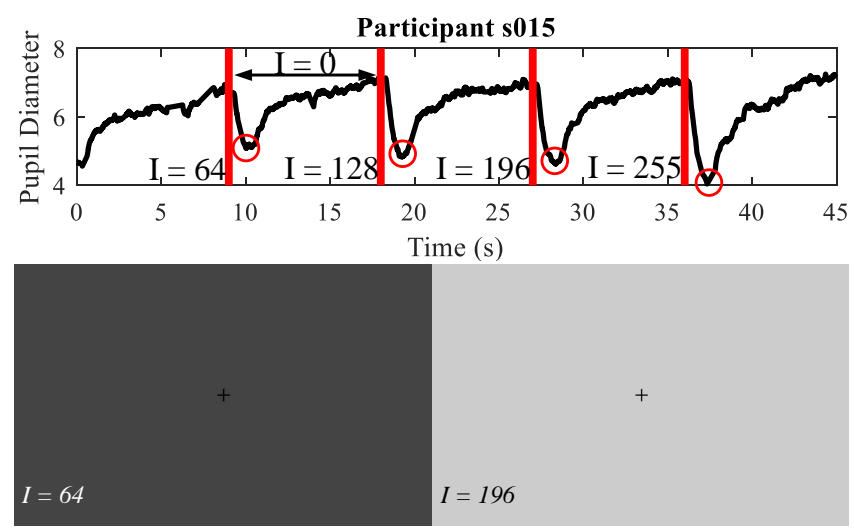

Figure 1: Top: Pupillary data for participant s015 performing a 2D PLR calibration. Solid red lines indicate when a grayscale intensity was presented, and red circles indicate the extracted pupil diameter associated with each intensity. Bottom: Grayscale intensity images for $I=64$ and $I=196$.

\section{METHODOLOGY}

\subsection{PLR Modelling}

For the PLR calibration I am using an approach proposed by [Raiturkar et al. 2016b], that displays a range of grayscale intensities on screen while recording pupil diameter. The grayscale image appears for a duration of one second, and then the screen returns to black for eight seconds. Figure 1 shows pupil diameter during the calibration procedure, and example stimuli.

Once this calibration has been performed there is a one to one mapping between pupil diameter in millimeters, and grayscale intensity in the range [0,255]. [Watson and Yellott 2012] provide a review of the models commonly used to predict pupil diameter from PLR, including linear, cubic, and exponential models. The model is used to compute the pupil diameter from PLR, $P D_{P L R}$; which is then subtracted from the recorded values during an experiment to generate a light compensated pupil diameter, $P D_{E}=P D-P D_{P L R}$, where $P D$ is the pupil diameter after pre-processing.

\subsection{User Studies \& Classification}

Computing $P D_{E}$ is straightforward after a calibration. Classifying these values to a specific state, i.e. aroused or sick, is a not as straightforward. One simple approach is compare the average $P D_{E}$ over a time window to an average baseline value computed during a neutral part of the experiment [Krejtz et al. 2018].

To branch into a more complex understanding of pupillary data, I propose correlating $P D_{E}$ with ground truth measures of arousal and simulator sickness in VR. An initial user study, $S_{1}$, would be conducted to correlate this feature with self reports provided during, and after the VR experience. Self reports will take the form of Likert style questionnaire responses and real-time feedback from a joystick used to report arousal and valence [Cowie et al. 2000; Mavridou et al. 2018]. There are three independent variables: the VR format, emotional arousal/valence, and level of motion inducing sickness.

A second study, $S_{2}$, would then be designed based on findings from the initial data collection. The goal of this study is to make the classification system more robust. This study would integrate

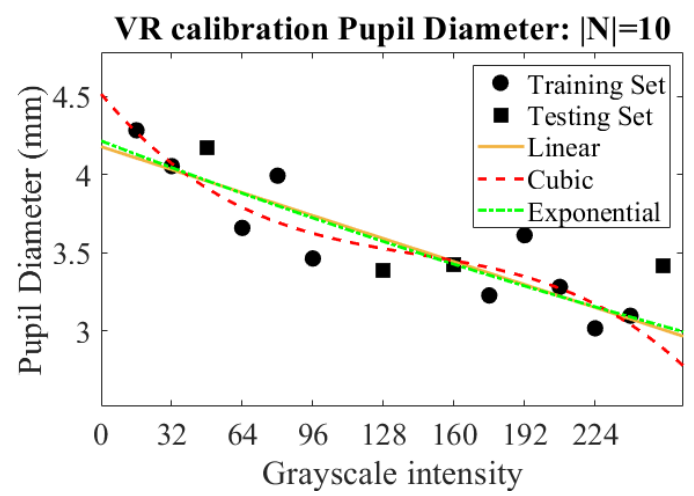

Figure 2: PLR models from one participant (s003) in VR.

additional physiological sensors into the data collection, removing the task of reporting real-time emotional feedback from the user. It is hypothesized that the reporting adds a significant amount of cognitive load and mental processing to the user [Malandrakis et al. 2011; Yannakakis and Martinez 2015].

A variety of models and machine learning techniques have been applied with similar data, including linear discriminants [Klotzsche et al. 2018], SVMs [Ramirez and Vamvakousis 2012], LSTM networks [Hofmann et al. 2018], and decision trees [Padmanaban et al. 2018]. The performance of machine learning models and feature selection techniques, such as PCA and random forests, will highlight how pupil diameter is linked to emotion and simulator sickness.

\section{PRELIMINARY RESULTS}

Preliminary work conducted thus far is concerned with $R_{1}$, and answering $H_{1}$. I worked to extend the calibration procedure described in Section 4.1 to a VR HMD, while increasing the number of intensity values from eight to sixteen and tested the effect of varying the order in which intensities were presented [John et al. 2018]. Linear, cubic, and exponential models were fit for each for each participant. Figure 2 shows one such model. We found that the exponential model performed best, which is in contrast to the 2D viewing scenario where the linear model performed best. We also found that the predictive error from the VR calibration was on the same scale as that of the 2D calibration, with an average error of $0.27 \mathrm{~mm}$. This has confirmed $H_{1}$, but there is still a need to evaluate these models at different depths.

\section{FUTURE WORK}

There are two components planned for future work. First, I must continue exploring $R_{1}$, and confirm if $H_{2}$ is true. If $H_{2}$ holds, we can proceed with exploring $R_{2}$. Otherwise, the model will have to be modified to account for depths at multiple stimuli. I would then use a calibration performed at different depths, and integrate the current gaze depth into the PLR model to predict pupil diameter from depth and light intensity.

Once $H_{1}$ and $H_{2}$ have been answered I will proceed with conducting studies $S_{1}$ and $S_{2}$. I already have access to a VR HMD with integrated eye tracker and a device to compute HRV and GSR (as described in [Raiturkar et al. 2016a]). The ultimate goal of my work is to produce a system that can use pupil diameter to predict emotional state or motion sickness in VR. 


\section{REFERENCES}

Jackson Beatty. 1982. Task-evoked pupillary responses, processing load, and the structure of processing resources. Psychological bulletin 91, 2 (1982), 276.

Margaret M Bradley and Peter J Lang. 1994. Measuring emotion: the self-assessment manikin and the semantic differential. Journal of behavior therapy and experimental psychiatry 25, 1 (1994), 49-59.

Margaret M Bradley, Laura Miccoli, Miguel A Escrig, and Peter J Lang. 2008. The pupil as a measure of emotional arousal and autonomic activation. Psychophysiology 45 , 4 (2008), 602-607.

Roddy Cowie, Ellen Douglas-Cowie, Susie Savvidou*, Edelle McMahon, Martin Sawey, and Marc Schröder. 2000. 'FEELTRACE': An instrument for recording perceived emotion in real time. In ISCA tutorial and research workshop (ITRW) on speech and emotion.

SG De Groot and JW Gebhard. 1952. Pupil size as determined by adapting luminance. FOSA 42, 7 (1952), 492-495.

Simon M Hofmann, Felix Klotzsche, Alberto Mariola, Vadim V Nikulin, Arno Villringer, and Michael Gaebler. 2018. Decoding subjective emotional arousal during a naturalistic VR experience from EEG using LSTMs. In 2018 IEEE International Conference on Artificial Intelligence and Virtual Reality (AIVR). IEEE, 128-131.

Brendan John, Pallavi Raiturkar, Arunava Banerjee, and Eakta Jain. 2018. An evaluation of pupillary light response models for 2D screens and VR HMDs. In Proceedings of the 24th ACM Symposium on Virtual Reality Software and Technology. ACM, 19.

Robert S Kennedy, Norman E Lane, Kevin S Berbaum, and Michael G Lilienthal. 1993. Simulator sickness questionnaire: An enhanced method for quantifying simulator sickness. The international journal of aviation psychology 3, 3 (1993), 203-220.

Felix Klotzsche, Alberto Mariola, Simon Hofmann, Vadim V Nikulin, Arno Villringer and Michael Gaebler. 2018. Using EEG to decode subjective levels of emotional arousal during an immersive VR roller coaster ride. In 2018 IEEE Conference on Virtual Reality and 3D User Interfaces (VR). IEEE, 605-606.

Krzysztof Krejtz, Andrew T Duchowski, Anna Niedzielska, Cezary Biele, and Izabela Krejtz. 2018. Eye tracking cognitive load using pupil diameter and microsaccades with fixed gaze. PloS one 13, 9 (2018), e0203629.

Nikos Malandrakis, Alexandros Potamianos, Georgios Evangelopoulos, and Athanasia Zlatintsi. 2011. A supervised approach to movie emotion tracking. In 2011 IEEE international conference on acoustics, speech and signal processing (ICASSP). IEEE, 2376-2379.

Sandra P Marshall. 2002. The index of cognitive activity: Measuring cognitive workload In Human factors and power plants, 2002. proceedings of the 2002 IEEE 7th conference on. IEEE, 7-7.

Ifigeneia Mavridou, Ellen Seiss, Theodoros Kostoulas, Charles Nduka, and Emili Balaguer-Ballester. 2018. Towards an effective arousal detection system for virtual reality. In Proceedings of the Workshop on Human-Habitat for Health (H3): HumanHabitat Multimodal Interaction for Promoting Health and Well-Being in the Internet of Things Era. ACM, 4.

Nitish Padmanaban, Timon Ruban, Vincent Sitzmann, Anthony M Norcia, and Gordon Wetzstein. 2018. Towards a machine-learning approach for sickness prediction in 360 stereoscopic videos. IEEE transactions on visualization and computer graphics 24, 4 (2018), 1594-1603.

Marco Pedrotti, Mohammad Ali Mirzaei, Adrien Tedesco, Jean-Rémy Chardonnet, Frédéric Mérienne, Simone Benedetto, and Thierry Baccino. 2014. Automatic stress classification with pupil diameter analysis. International fournal of HumanComputer Interaction 30, 3 (2014), 220-236.

Pallavi Raiturkar, Susan Jacobson, Beida Chen, Kartik Chaturvedi, Isabella Cuba, Andrew Lee, Melissa Franklin, Julian Tolentino, Nia Haynes, Rebecca Soodeen, et al. 2016a. Measuring viewers' heart rate response to environment conservation videos. In Proceedings of the ACM Symposium on Applied Perception. ACM, 138-138.

Pallavi Raiturkar, Andrea Kleinsmith, Andreas Keil, Arunava Banerjee, and Eakta Jain 2016b. Decoupling light reflex from pupillary dilation to measure emotional arousal in videos. In Proceedings of the ACM Symposium on Applied Perception. ACM, 89-96.

Rafael Ramirez and Zacharias Vamvakousis. 2012. Detecting emotion from EEG signals using the emotive epoc device. In International Conference on Brain Informatics. Springer, 175-184.

Prentice Reeves. 1918. Rate of pupillary dilation and contraction. Psychological Review 25, 4 (1918), 330.

Andrew B Watson and John I Yellott. 2012. A unified formula for light-adapted pupil size. Fournal of Vision 12, 10 (2012), 12-12.

Olivier White and Robert M French. 2017. Pupil diameter may reflect motor control and learning. Fournal of motor behavior 49, 2 (2017), 141-149.

Georgios N Yannakakis and Hector P Martinez. 2015. Grounding truth via ordinal annotation. In 2015 international conference on affective computing and intelligent interaction (ACII). IEEE, 574-580.

Francis A Young and William R Biersdorf. 1954. Pupillary contraction and dilation in light and darkness. Journal of comparative and physiological psychology 47, 3 (1954), 264 